\title{
Effects of initial state nucleon shadowing on the elliptic flow of thermal photons
}

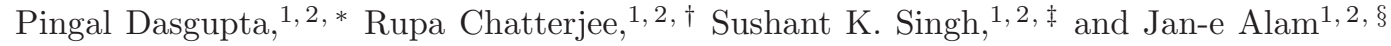 \\ ${ }^{1}$ Variable Energy Cyclotron Centre, 1/AF, Bidhan Nagar, Kolkata-700064, India \\ ${ }^{2}$ Homi Bhabha National Institute, Training School Complex, Anushakti Nagar, Mumbai 400085, India
}

\begin{abstract}
Recently the effect of nucleon shadowing on the Monte-Carlo Glauber initial condition was studied and its role on the centrality dependence of elliptic flow $\left(v_{2}\right)$ and fluctuations in initial eccentricity for different colliding nuclei were explored. It was found that the results with shadowing effects are closer to the QCD based dynamical model as well as to the experimental data. Inspired by this outcome, in this work we study the transverse momentum $\left(p_{T}\right)$ spectra and elliptic flow of thermal photons for $\mathrm{Au}+\mathrm{Au}$ collisions at $\mathrm{RHIC}$ and $\mathrm{Pb}+\mathrm{Pb}$ collisions at LHC by incorporating the shadowing effects in deducing the initial energy density profile required to solve the relativistic hydrodynamical equations. We find that the thermal photon spectra remain almost unaltered, however, the elliptic flow of photon is found to be enhanced significantly due to shadowing effects.
\end{abstract}

\section{INTRODUCTION}

The primary goal of heavy ion collision programmes at Relativistic Heavy Ion Collider (RHIC) and Large Hadron Collider (LHC) is to produce a new state of thermalized matter called quark gluon plasma (QGP) - where the properties of the system are not governed by hadrons but by the quarks and gluons. Collision of nuclei will create charged particles either in the form of hadrons or partons depending on the magnitude of the collision energy. Electromagnetic interaction among these charged particles will inevitably lead to the production of photons with mean free path much larger than the size of the system formed in such collisions. As a result photons can bring the information of the production point very efficiently without getting affected by the secondary interactions and therefore, considered as one of the efficient probes for the detection of QGP [1]. This has led to huge theoretical 22 12] and experimental [13 15] efforts to study the mechanism of photon production in heavy ion collisions at relativistic energies (HICRE). Recent data from PHENIX [14] and ALICE [15] Collaborations at the RHIC and LHC respectively have reported excess of direct photons over the scaled yield from protonproton collisions in the transverse momentum domain, $p_{T}<4 \mathrm{GeV}$. This excess is attributed to the thermal radiation from QGP and hadronic matter. However, in spite of a large number of detailed studies by several authors [16 20] a simultaneous explanation of the data on $p_{T}$ spectra and differential elliptic flow of photons is still lacking both at RHIC [21] and at the LHC 22] energies, the lack of this explanation has been dubbed as "direct photon puzzle" [23].

The QGP produced in heavy ion collisions at RHIC and LHC energies evolve in space and time and revert to hadrons at transition temperature, $T_{c}$. In such a sce-

\footnotetext{
*Electronic address: pingaldg@vecc.gov.in

${ }^{\dagger}$ Electronic address: rupa@vecc.gov.in

$\ddagger$ Electronic address: sushantsk@vecc.gov.in

$\S$ Electronic address: jane@vecc.gov.in
}

nario photons will be produced through various processes at different stages of the evolution populating different domains of $p_{T}$. These are broadly categorized as: (i) prompt photons originating from the interactions of the partons of the colliding nuclei which will populate the high $p_{T}$ domain; (ii) thermal productions - from the interactions of thermal partons as well as from thermal hadrons - will occupy low and intermediate $p_{T}$ and finally (iii) from the decays of the long lived (compared to strong interaction time scale) mesons. Photons from (i) are non-thermal therefore, are affected neither by the temperature nor by the flow. For a given collision energy this contribution could be subtracted out from the data by using $\mathrm{pQCD}$ results. Photon spectra from proton+proton collision may be used as a benchmark to validate theoretical results. Photons from the decays of hadrons have been subtracted out in the data presented by ALICE and PHENIX Collaborations [14, 15]. Photons from thermal source, (ii) are sensitive to temperature and flow, therefore, this is the component of the spectra that needs urgent attention to address the "direct photon puzzle".

Elliptic flow is considered as one of the fundamental observables of collectivity of the system produced in HICRE. Thermal photons originating from expanding QGP along with prompt photons explain the data on the photon spectra both for $\mathrm{Au}+\mathrm{Au}$ collisions at RHIC and $\mathrm{Pb}+\mathrm{Pb}$ collisions at $\mathrm{LHC}$ energies in the region $p_{T}>$ $2 \mathrm{GeV}[24,25]$. However, the large discrepancies between theoretical results and the data on thermal photon's $v_{2}$ remains a puzzle. Several studies such as calculations based on sophisticated event-by-event viscous hydrodynamic model, studies incorporating pre-equilibrium contributions and including the effects of high initial magnetic field etc. are unable to explain the data on $v_{2}$ of photons till date.

With the increase in the precision of experimental measurements, it becomes imperative for theoretical calculations to include finer physical effects. One such effect is shadowing of nucleons deep inside the colliding nuclei by the nucleons at the front during the process of collisions. In an effort to understand the correlation between 
the multiplicity and eccentricity, effect of shadowing was included in Refs. [26, 27] in the Monte-Carlo Glauber model to deduce the initial energy density profile which is required as an input to the relativistic hydrodynamical equations. Solutions of relativistic hydrodynamical equations are used to understand the development of collectivity in the system. It was observed that the inclusion of shadowing in the Monte-Carlo (MC) Glauber model increases the elliptic flow significantly compared to the result obtained from the conventional MC Glauber initial condition. In this paper, we explore the effects of nucleon shadowing on the elliptic flow of photons at RHIC and LHC energies.

We set the goal of the paper at this point. In this work our aim is not to reproduce the experimental data on $v_{2}$ and thermal spectra of photons or to solve the "direct photon puzzle" but to estimate $v_{2}$ by including the effects of shadowing that a nucleon deep inside the colliding nucleus is subjected to. In this context we evaluate $v_{2}$ and thermal spectra with and without shadowing effects by fixing the value of charged hadron multiplicity to its experimental value.

The paper is organized as follows. In the next section we will discuss the MC Glauber model with and without shadowing effects. Production mechanisms of photons from QGP and HM have been briefly discussed in section III with appropriate references for details. The effects of shadowing on the hydrodynamic evolution and elliptic flow of thermal photon spectra have been presented in section IV. Section V is devoted to summary and conclusions.

\section{INITIAL CONDITIONS}

In the conventional MC Glauber model, all the nucleons are given equal weightage for energy deposition i.e. a nucleon undergoing multiple collisions will deposit the same amount of energy in each collision. In Ref. [26] it is argued that the nucleons located deep inside the nucleus are eclipsed or shadowed by the nucleons in the front. Therefore, the contribution of a participating nucleon to energy deposition will crucially depend on its position in the colliding nuclei. This is accomplished by introducing a weight factor $S(n, \lambda)$ in the initial state as follows:

$$
S(n, \lambda)=\exp (-n \lambda)
$$

where, $S(n, \lambda)$ accounts for shadowing on a participant due to $n$ other nucleons in the same nucleus which are in front and conceal it partially (see Refs. [26, 27] for more detail). In the present work we took $\lambda=0.12$ and 0.08 at RHIC and LHC respectively [26, 27]. We call the MC Glauber initial condition without the shadowing effect as MCG and the initial condition with the shadowing as shMCG.

A MCG model with standard two-parameter WoodsSaxon nuclear density profile is used to randomly distribute the nucleons into the two colliding nuclei. Two nucleons from different nuclei are assumed to collide when the relation $d^{2}<\frac{\sigma_{N N}}{\pi}$ is satisfied where, $d$ is the transverse distance between the colliding nucleons and $\sigma_{N N}$ is the inelastic nucleon-nucleon cross-section. We take $\sigma_{N N}$ as $42 \mathrm{mb}$ and $64 \mathrm{mb}$ for $200 \mathrm{~A} \mathrm{GeV} \mathrm{Au}+\mathrm{Au}$ collision at $\mathrm{RHIC}$ and $2.76 \mathrm{~A} \mathrm{TeV} \mathrm{Pb}+\mathrm{Pb}$ collision at $\mathrm{LHC}$ respectively.

For the collision of two nuclei, we assume the beam axis to be along $z$-direction and impact parameter to be along $x$-direction for a particular event. The plane spanned by the $x$ - and $y$-axes is the transverse plane. The initial entropy distribution, $s(x, y)$, is obtained by first locating $(x, y)$-coordinates of participants and binary collisions which are treated as sources of energy deposition, and then taking a weighted sum over all the sources as explained in Eq. 2 below. The coordinates of a binary collision is taken as the average of the coordinates of two colliding nucleons. Hence, sources are distributed randomly in the transverse plane and each source receives a different weight for energy deposition depending on whether the source is a participant or a binary collision. For MCG, a participant is given a weight $(1-\nu)$ and a binary collision is given a weight of $\nu$, where $\nu$ is some constant which is obtained by fitting with the experimental data of charged hadron multiplicity distribution $\left(d N_{\mathrm{ch}} / d \eta\right)$. The entropy density is then obtained using the following expression,

$$
s(x, y)=K \sum_{i=1}^{N_{s}} w_{i}\left(\Theta_{i}\right) f_{i}(x, y)
$$

where $\Theta_{i}$ is a binary variable used to label the source and which decides the type of weight to be given making $w_{i}$ a function of $\Theta_{i}$ which is denoted by $w_{i}\left(\Theta_{i}\right)$. We take $w_{i}=\nu$ and $(1-\nu)$ for $\Theta_{i}=0$ and $\Theta_{i}=1$ respectively. In Eq. 2, $N_{s}$ denotes the total number of sources and $f_{i}(x, y)$ is the normalized distribution given by,

$$
f_{i}(x, y)=\frac{1}{2 \pi \sigma^{2}} e^{-\frac{\left(x-x_{i}\right)^{2}+\left(y-y_{i}\right)^{2}}{2 \sigma^{2}}} .
$$

Note that in the limit $\sigma \rightarrow 0, \mathrm{Eq} 2$ reduces to the two component formula :

$$
s(x, y)=K\left[\nu n_{\text {coll }}(x, y)+(1-\nu) n_{\text {part }}(x, y)\right]
$$

where $n_{\text {coll }}$ and $n_{\text {part }}$ are number of collisions and number of participants respectively. The form of entropy density in shMCG is same as in Eq. 2 except that the weights $w_{i}$ are different. For $\Theta_{i}=0, w_{i}=\nu S_{i}^{c}$ and for $\Theta_{i}=1, w_{i}=(1-\nu) S_{i}^{p}$ where $S_{i}^{p}$ and $S_{i}^{c}$ are the shadowing weights. $S_{i}^{p}$ is given by Eq. 1, denotes weight factor when a participant nucleon is subjected to due to shadowing. To obtain the shadowing weights, we follow Ref [26]. Each nucleon is first assigned a weight depending on how many other nucleons are in front. $S_{i}^{p}$ is then the weight of the corresponding wounded nucleon and $S_{i}^{c}$ is the product of weights of the nucleons undergoing the collision. In Eq. 3, $\sigma$ is a free parameter, taken as $\sigma=$ $0.4 \mathrm{fm}$ [24, 28] for both MCG and shMCG. 
We take the values of initial thermalization times for RHIC and LHC as $\tau_{0}=0.17 \mathrm{fm} / c$ and $0.14 \mathrm{fm} / \mathrm{c}$ respectively from the EKRT mini-jet saturation model [29]. In the present work, $(2+1)$ dimensional inviscid relativistic hydrodynamic model has been used to study the spacetime evolution of the matter produced in HICRE [28]. Same hydrodynamical model with MCG initial condition has been used earlier to study spectra and anisotropic flow of hadrons and photons [24, 25, 28]. We modify the initial conditions to include the shadowing effect.

The initial entropy density profile required as input to hydrodynamical calculation is constructed for both the cases (MCG and shMCG) by taking initial state average of $N(=10000)$ random events (where events obey the distribution $d N / d b \propto b, b$ is impact parameter) within the particular centrality class as follows :

$$
s(x, y)=\frac{1}{N} \sum_{i=1}^{N} s_{i}(x, y)
$$

where, $s_{i}(x, y)$ characterizes the entropy distribution of the $i^{t h}$ event produced according to the Eq. 2. The initial flow velocity component, $v_{x}$ and $v_{y}$ are taken as zero here.

\section{PHOTONS FROM THERMAL QGP AND HADRONS}

Contributions from the QGP matter to the thermal photon spectra due to annihilation $(q \bar{q} \rightarrow g \gamma)$ and the QCD Compton $(q(\bar{q}) g \rightarrow q(\bar{q}) \gamma)$ processes to the order $\alpha_{s} \alpha$ was estimated in [30, 31] by using hard thermal loop (HTL) approximation 32]. Later, it was shown that photons from the processes [33]: $g q \rightarrow g q \gamma, q q \rightarrow q q \gamma, q q \bar{q} \rightarrow q \gamma$ and $g q \bar{q} \rightarrow g \gamma$ contribute also to the same order as $O\left(\alpha \alpha_{s}\right)$. The complete calculation of emission rate from QGP to order $\alpha_{s}$ has been performed by resumming ladder diagrams in the effective field theory [34, 35]. In the present work, the rate of production of thermal photons has been taken from [34]. The $T$ dependence of the strong coupling, $\alpha_{s}$ has been taken from [36].

For the photon production from hadronic matter an exhaustive set of hadronic reactions and the radiative decays of resonances are considered. The rate has been taken from [37] which includes the effects of the hadronic form factor (see also 38 40]).

The $p_{T}$ distribution of photons is obtained by integrating the temperature dependent emission rates $(R=$ $\left.E d N / d^{3} p d^{4} x\right)$ over the entire space-time evolution history - from the initial thermalization time to the final freeze-out state of the fireball via intermediary quarkhadron transition as :

$$
\begin{aligned}
E \frac{d N}{d^{3} p}= & \int d^{4} x\left\{R_{Q}\left(E^{*}(x), T(x)\right) \Theta\left(T-T_{c}\right)\right. \\
& \left.+R_{H}\left(E^{*}(x), T(x)\right) \Theta\left(T_{c}-T\right) \Theta\left(T-T_{F}\right)\right\}(6)
\end{aligned}
$$

where, $T(x)$ is the local temperature and $E^{*}(x)=$ $p^{\mu} u_{\mu}(x)$ is the energy in the comoving frame, $p^{\mu}$ is the four-momentum of the photons and $u_{\mu}$ is the local fourvelocity of the flow field, $R_{Q}\left(R_{H}\right)$ is the emission rate of photons from QGP (hadronic system). $T$ and $u^{\mu}$ are obtained from the solution of hydrodynamical equations.

The elliptic flow parameter $v_{2}$ is calculated by using the relation :

$$
v_{2}\left(p_{T}\right)=\langle\cos (2 \phi)\rangle=\frac{\int_{0}^{2 \pi} d \phi \cos (2 \phi) \frac{d N}{p_{T} d p_{T} d y d \phi}}{\int_{0}^{2 \pi} d \phi \frac{d N}{p_{T} d p_{T} d y d \phi}} .
$$

The temperature at freeze-out is taken as $160 \mathrm{MeV}$ which reproduces the measured $p_{T}$ spectra of charged pions at RHIC and LHC energies. The value of quark-hadron transition temperature is taken as $170 \mathrm{MeV}$ and the lattice QCD based EoS is taken from [41] to solve the hydrodynamical equations.

\section{RESULTS}

We study the effects of shadowing by considering the following two cases:

Case-I : First we consider the case where $\nu=0$. This gives the wounded nucleon profile. We study the difference in the evolution scenario with and without shadowing effects. In the following, we calculate averages of hydrodynamic quantities by using the following relation:

$$
\langle f\rangle=\frac{\int \mathrm{d} x \mathrm{~d} y f(x, y) \epsilon(x, y, \tau)}{\int \mathrm{d} x \mathrm{~d} y \epsilon(x, y, \tau)}
$$

where, $\epsilon(x, y, \tau)$ is the energy density at $(x, y)$ at proper time, $\tau$ obtained by solving the hydrodynamic equations.

We construct the MCG initial condition by taking $K=102 \mathrm{fm}^{-1}$ in Eq. 2. The MCG initial condition has been used extensively earlier to calculate photon production at RHIC and LHC energies [24, 25]. The time evolution of average temperature, $\langle T\rangle$ and average transverse flow velocity, $\left\langle v_{T}\right\rangle$ for MCG initial condition for $200 \mathrm{~A}$ $\mathrm{GeV} \mathrm{Au}+\mathrm{Au}$ collisions at RHIC in $20-40 \%$ centrality bin are shown by black dashed lines in Fig. 1(a) and Fig. 1(b) respectively. The inclusion of initial state shadowing, i.e., $\lambda \neq 0$, in the MCG initial condition and without changing $K$ (from now on we call this as unmodified shMCG) results in smaller charged hadron multiplicity or total entropy. This leads to smaller average temperature (as shown by blue dotted lines in Fig. 1(a)) and pressure, which in turn results in smaller average transverse flow velocity (shown by blue dotted lines in Fig. 1(b)).

In order to obtain the initial condition for shMCG, the normalization factor $K$ (Eq. 2) is tuned to reproduce the same final $d N_{\mathrm{ch}} / d \eta$ as MCG initial condition. We obtain $K=140 \mathrm{fm}^{-1}$ for shMCG. The time evolution of $\langle T\rangle$ and $\left\langle v_{T}\right\rangle$ for the shMCG initial condition are shown by red solid lines in Fig. 1(a) and Fig. 1(b) respectively. It is to 

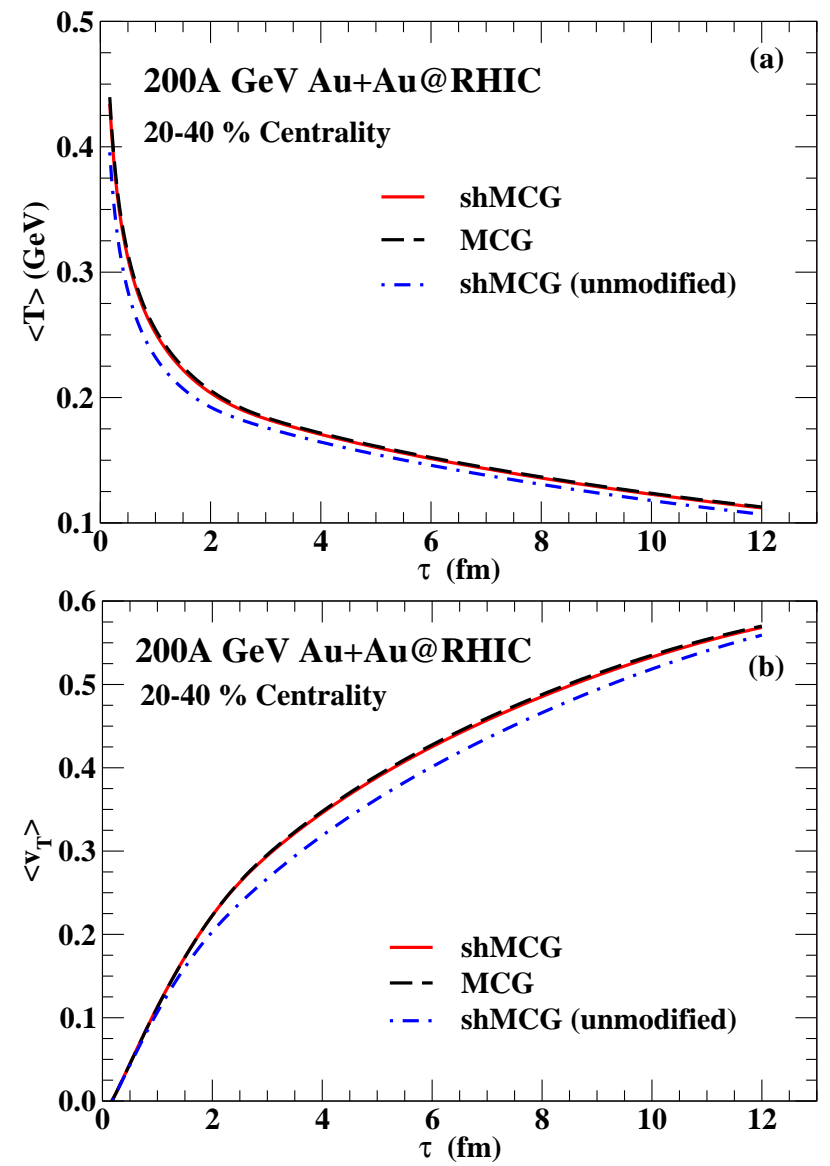

FIG. 1: (Color online) (a) Time evolution of average temperature and (b) transverse flow velocity with MCG and shMCG initial conditions have been depicted for $\nu=0$.

be noticed that the differences in $\langle T\rangle$ and $\left\langle v_{T}\right\rangle$ for MCG and shMCG are insignificant when the model parameters in shMCG are adjusted to reproduce the same charged hadron multiplicity as in MCG.

Next we display our results on the $p_{T}$ spectra of photons. Fig. 2(a) shows the thermal photon spectra with MCG and shMCG initial conditions for $20-40 \%$ central $\mathrm{Au}+\mathrm{Au}$ collisions at RHIC. For given production rate of photons with similar space-time evolution scenario, the spectra for the two initial conditions (MCG and shMCG) are found to be close to each other. For both the cases, photon spectra are dominated by radiation from the QGP for $p_{T}>1 \mathrm{GeV}$ and only in the low $p_{T}(<1 \mathrm{GeV})$ region we see significant contribution from the hadronic matter. However, for unmodified shMCG the spectra is found to be suppressed.

The elliptic flow of thermal photons calculated using MCG and shMCG initial conditions are shown in Fig. 2(b). As shown in earlier studies [25, 42], due to the competing contributions from the quark matter and hadronic matter (HM) states, the $v_{2}$ of photons shows different nature compared to that of hadrons.

The differential elliptic flow of photons is small at
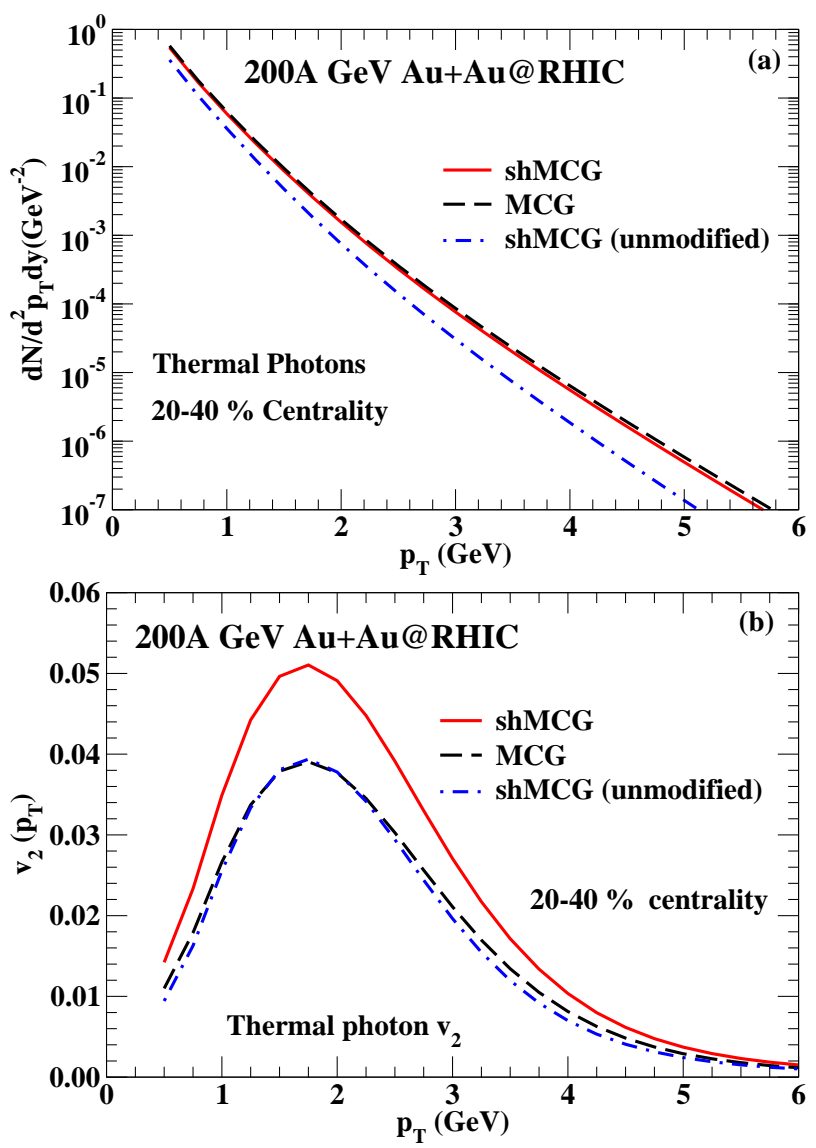

FIG. 2: (Color online) (a) Thermal photon spectra and (b) elliptic flow from MCG and shMCG initial conditions for wounded profile at RHIC $200 \mathrm{~A} \mathrm{GeV} \mathrm{Au+Au} \mathrm{collisions} \mathrm{in} \mathrm{20-}$ $40 \%$ centrality bin.

large $p_{T}$ as these are mostly emitted from the (early) QGP state where the flow has not developed fully. As we approach from high $p_{T}$, the $v_{2}\left(p_{T}\right)$ increases towards smaller values of $p_{T}$, reaches maximum around $1.5-2.0$ $\mathrm{GeV}$ and then drops as $p_{T}$ is reduced further. Such a variation of $v_{2}\left(p_{T}\right)$ results from the two competing profiles of elliptic flow originating from QGP and hadronic matter states (see later).

We see a significant increase in the elliptic flow for the shMCG initial condition compared to the MCG initial condition. The value of $v_{2}\left(p_{T}\right)$ at the peak is about $32 \%$ larger in the shMCG case. This may be qualitatively explained as follows. The inclusion of shadowing in the initial condition affects the nucleons situated in the interior of nucleus more strongly than those at the boundary [26] . This means that the shadowing effects are less prominent at the ends of the major axis of elliptic overlap zone in the transverse plane where the nucleons from the boundary of the two nuclei are involved. Whereas, the shadowing effects are more prominent at the ends of the minor axis where nucleons from the interior of one of the nuclei with large shadowing effects collide with the nucleons from the boundary of the other nucleus. This results in 

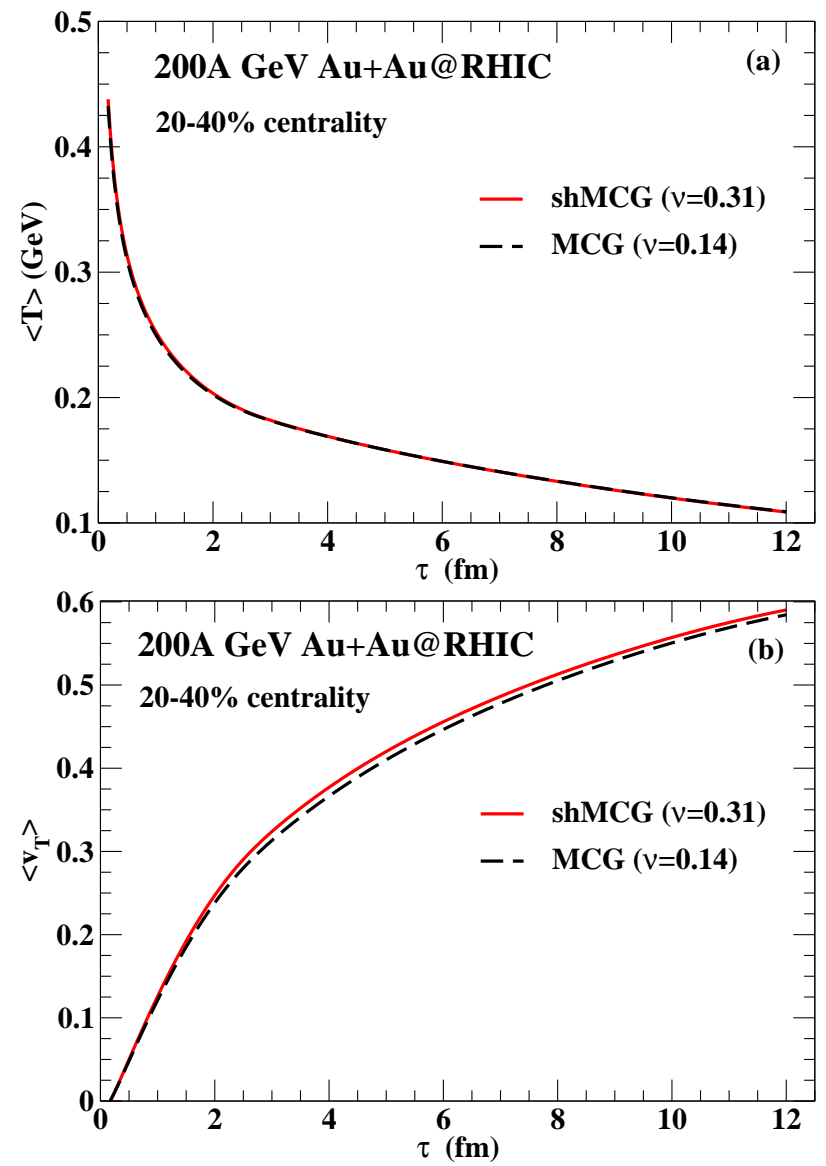

FIG. 3: (Color online) (a) Time evolution of average transverse flow velocity and (b) average temperature considering MCG and shMCG initial states for two component model $(\nu \neq 0)$.

smaller effective length of the minor axis in shMCG than MCG, as a consequence the pressure gradient in shMCG is larger resulting in larger elliptic flow. Interestingly, one can see that the $v_{2}$ from the unmodified shMCG initial condition (in which shadowing is included but $K$ is not fixed to reproduce the final particle multiplicity) is close to the $v_{2}$ from MCG initial condition even when shadowing introduces more spatial anisotropy because of the lower pressure (originating from smaller hadronic multiplicity or entropy density) in case of unmodified shMCG scenario (Fig. 1 (b)).

In the present study we consider initial state averaged smooth density distribution and show that even though the photon spectra remain unaltered, the effect of shadowing on the elliptic flow is significantly large. It has been shown earlier that event-by-event fluctuating initial conditions increase $v_{2}$ significantly in the region $p_{T}>2$ $\mathrm{GeV}$ compared to the $v_{2}$ calculated from a smooth initial state profile [4]. Thus, result from calculation based on event-by-event fluctuating initial condition with shadowing is expected to enhance the $v_{2}$ even more. This would reduce the difference between the experimental data and

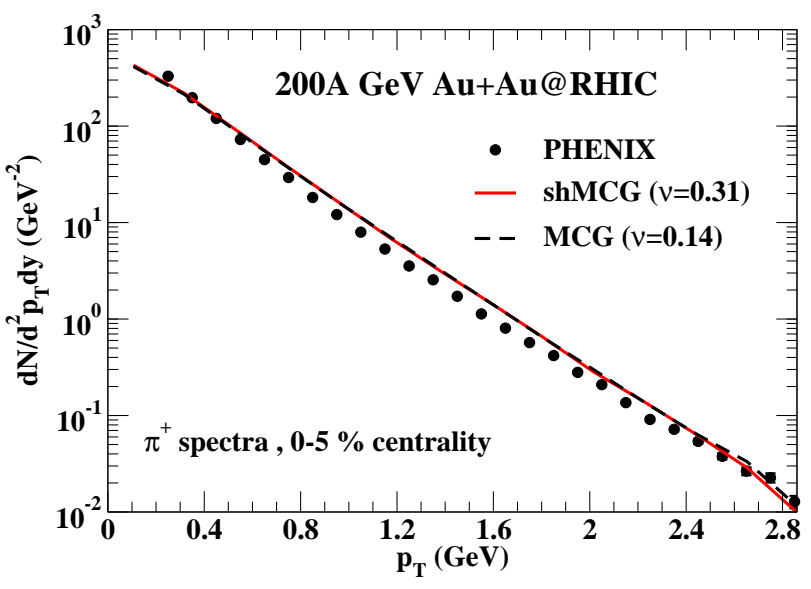

FIG. 4: (Color online) The thermal pion spectra at RHIC with two types of initial condition (see text) and comparison with PHENIX data [45].

theoretical results and would help in resolving the "direct photon puzzle". In addition, the triangular flow of photons $\left(v_{3}\right)$ which originates from the initial state fluctuations only, is also expected to be larger using shMCG initial condition. These aspects of study is postponed for future [43].

Case-II : Next we consider the case $\nu \neq 0$. The nucleon shadowing affects $N_{\text {coll }}$ more strongly than $N_{\text {part }}$ as shown in Ref. [26]. Therefore, we consider $\mathrm{Au}+\mathrm{Au}$ collisions at RHIC where the initial entropy density is taken as proportional to a linear combination of $N_{\text {coll }}$ and $N_{\text {part }}$. We take $\nu=0.14, K=80 \mathrm{fm}^{-1}$ for the MCG initial state and $\nu=0.31$ and $K=110 \mathrm{fm}^{-1}$ for shMCG. It may be noted here that different values of $K$ and $\nu$ in shMCG are required to reproduce the same charged hadron multiplicity measured. The transverse momentum spectra of $\pi^{+}$has been evaluated including the feed-down from higher resonance decays at the freeze-out surface by using Cooper-Frye 44] formula. The result has been contrasted with experimental data from RHIC [45]. We find that the $p_{T}$ spectra for $\pi^{+}$for MCG and shMCG are similar and both are close to experimental data (Fig. 4).

The time evolution of $\langle T\rangle$ and $\left\langle v_{T}\right\rangle$ for two initial conditions (MCG and shMCG) are shown in Fig. 3. The $\langle T\rangle$ for the two initial conditions are almost on top of each other whereas, the $\left\langle v_{T}\right\rangle$ is found to be marginally larger for shMCG initial condition.

For $\nu \neq 0$, successive collisions are allowed as opposed to the case with $\nu=0$. Therefore, amount of energy deposition is different for $\nu=0$ and $\nu \neq 0$ as large shadowing will take place in the latter case. The pressure gradient in shMCG with $\nu \neq 0$ is larger compared to the case where $\nu=0$. As a consequence the $\left\langle v_{T}\right\rangle$ in shMCG with $\nu \neq 0$ is slightly larger compared to the case where $\nu=0$.

The spectra and elliptic flow of thermal photons for $\nu \neq 0$ are shown in Fig. 5. (a) and Fig. 5. (b) respectively. The nature of the $p_{T}$ spectra and elliptic flow is found 

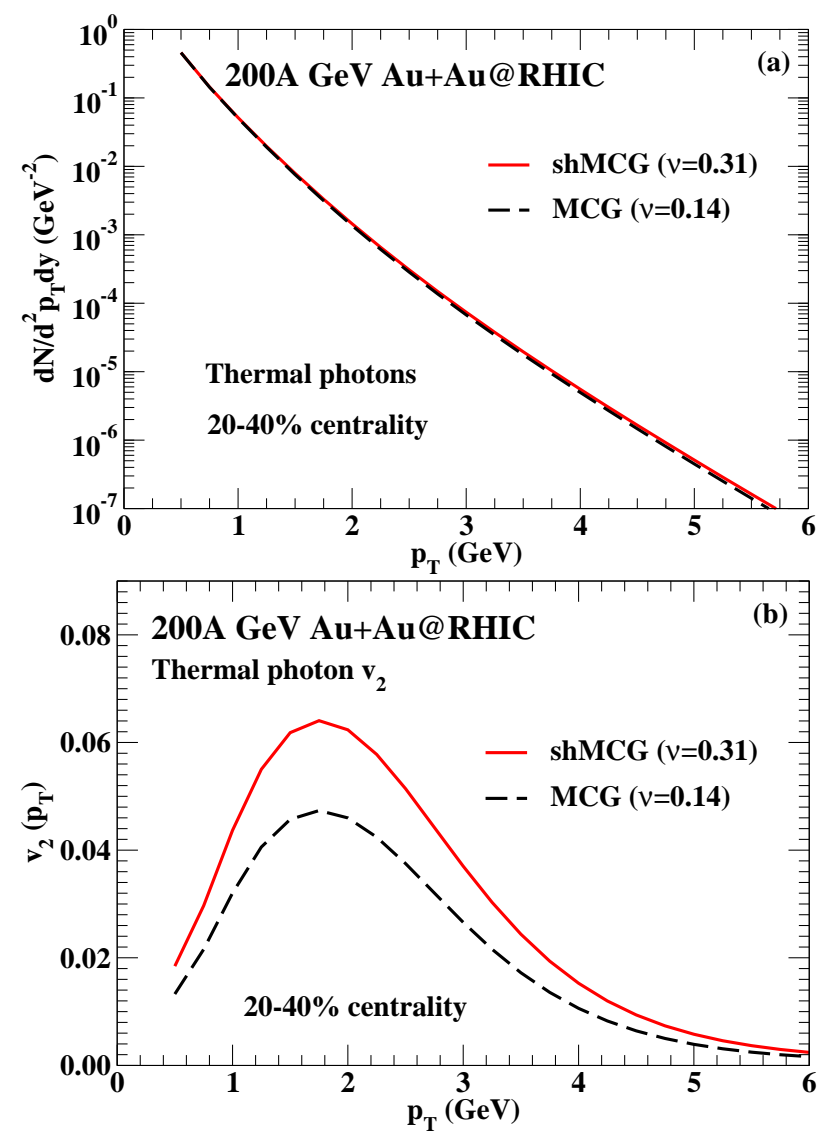

FIG. 5: (Color online) (a) Thermal photon spectra and (b) elliptic flow considering MCG and shMCG initial conditions for $200 \mathrm{~A} \mathrm{GeV} \mathrm{Au}+\mathrm{Au}$ collisions at RHIC and $20-40 \%$ centrality bin for two component model.

to be similar to the results from wounded nucleon profile. As shown in the figure, the $p_{T}$ spectra for MCG and shMCG initial conditions are again found to be close to each other. However, the elliptic flow is slightly larger for two component initial conditions compared to the $v_{2}$ from corresponding single component $(\nu=0)$ model. The peak value of $v_{2}\left(p_{T}\right)$ for shMCG initial condition is about $36 \%$ larger than the $v_{2}\left(p_{T}\right)$ calculated using the MCG initial condition. As explained before the effects of shadowing in the region near the ends of the major axis are smaller than region near ends of the minor axis of the elliptic overlap zone, which effectively reduces the minor to major axis ratio in shMCG compared to MCG resulting in larger elliptic flow in the shMCG scenario.

In order to understand the effect of initial state shadowing on the photon elliptic flow better, we plot the spectra and elliptic flow from individual QGP and HM separately in Fig. 6. The thermal photon spectra as expected from both QGP and HM are close to each other for the two different initial conditions as shown in Fig.6(a). The effect of initial state shadowing is found to be more pronounced for $v_{2}$ of photons from QGP (Fig. 6(b)) compared to $v_{2}$ from HM (Fig. 6 (c)) in the region $p_{T} \sim 1.5$
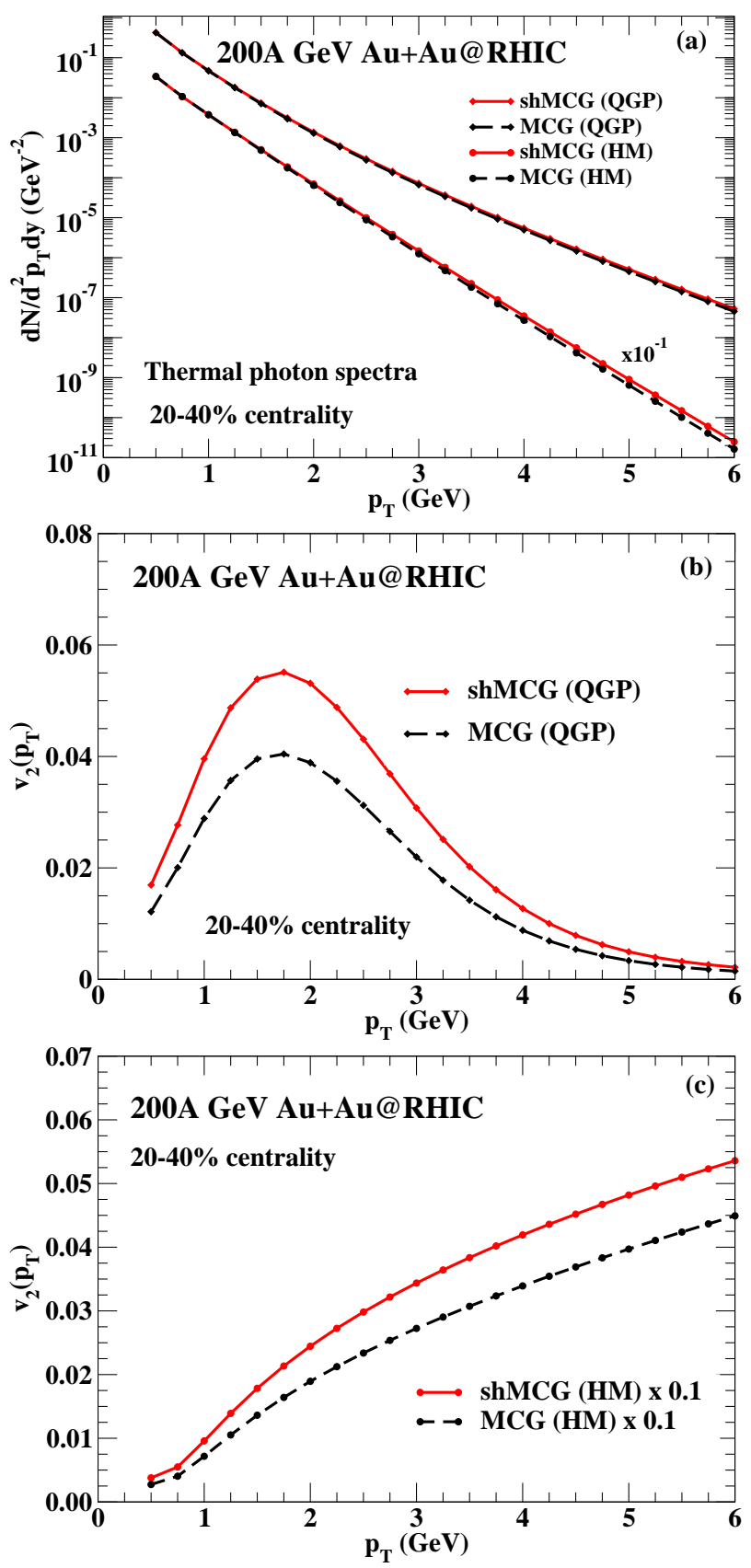

FIG. 6: (Color online) (a) Thermal photon spectra and elliptic flow from (b) QGP and (c) HM separately from MCG and shMCG initial conditions.

to $2.0 \mathrm{GeV}$ (where the increase in total photon $v_{2}$ due to initial state shadowing is maximum, Fig. 河). The $v_{2}\left(p_{T}\right)$ is about $50 \%$ larger in this region for shMCG compared to the MCG initial condition in the QGP, whereas the increase due to shMCG in HM is about $30 \%$.

Next we consider $\mathrm{Pb}+\mathrm{Pb}$ collision at $2.76 \mathrm{~A} \mathrm{TeV}$ energy at the LHC. We evaluate the $p_{T}$ spectra of thermal photons and elliptic flow using MCG and shMCG initial conditions.

We take $\nu=0.11, K=192 \mathrm{fm}^{-1}$ for the MCG ini- 

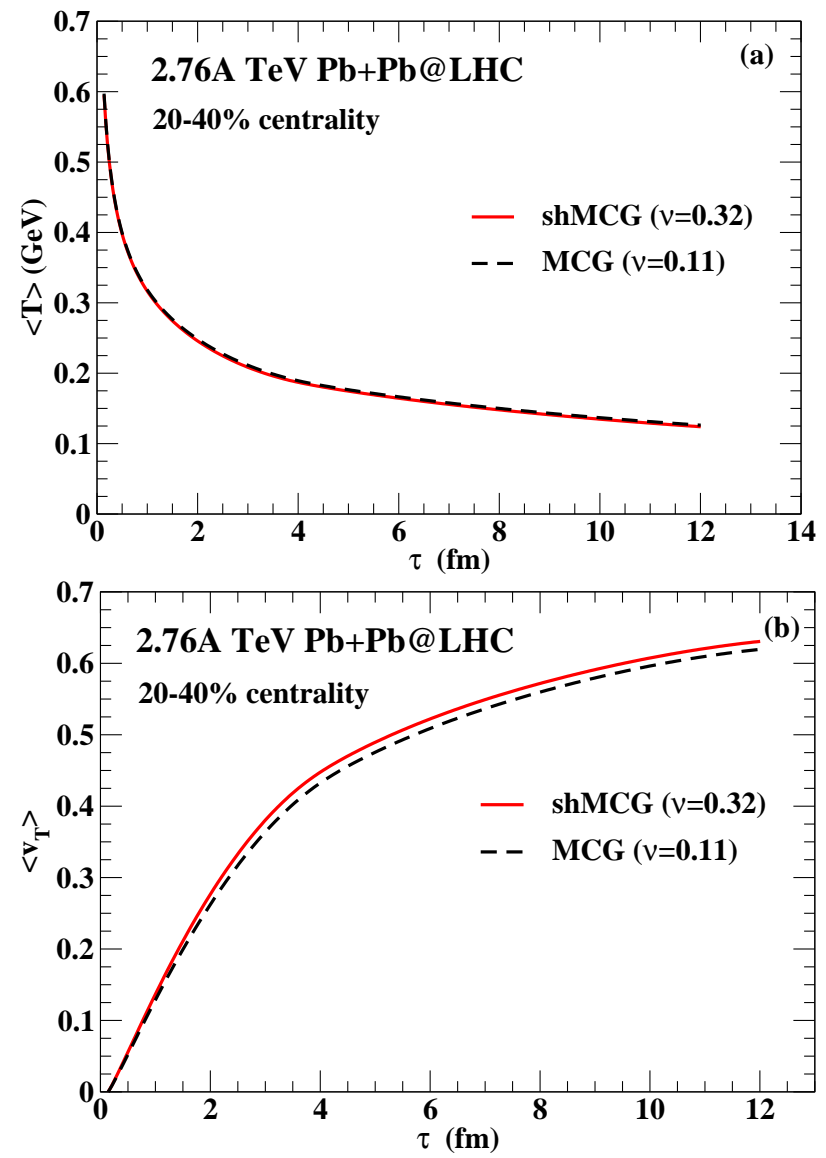

FIG. 7: (Color online) (a) The time evolution of temperature and (b) transverse velocity estimated for LHC energy with two types of initial conditions (see text).

tial state and $\nu=0.32, K=223 \mathrm{fm}^{-1}$ for the shMCG initial condition to reproduce the same charged hadron multiplicity at LHC. In Fig. 7 we display the variation of average temperature and transverse velocity with $\tau$ for LHC energy. Although, the results are qualitatively similar to RHIC, however, quantitatively the values of $\langle T\rangle$ and $\left\langle v_{T}\right\rangle$ are larger at LHC because of the larger initial temperature and pressure of the system created in $\mathrm{Pb}+\mathrm{Pb}$ collisions.

The $\pi^{+}$spectra for LHC collision condition have been evaluated at the freeze-out surface and the results are compared with the experimental data [46]. The spectra from MCG and shMCG are found to be close to each other (Fig. 8).

Finally, in Fig. 9 the transverse momentum spectra and $v_{2}$ of photons have been depicted for LHC collision condition. The effects of shadowing do not show up in the $p_{T}$ spectra of photons (Fig. 9(a)). However, the elliptic flow (Fig. 9(b)) is about $50 \%$ enhanced at the peak value in shMCG as compared to MCG for reasons explained above.

In order to get some idea about the effect of shadowing on hadronic observables, we calculate the differential elliptic flow of $\pi^{+}$for RHIC (Fig. 10(a)) and LHC

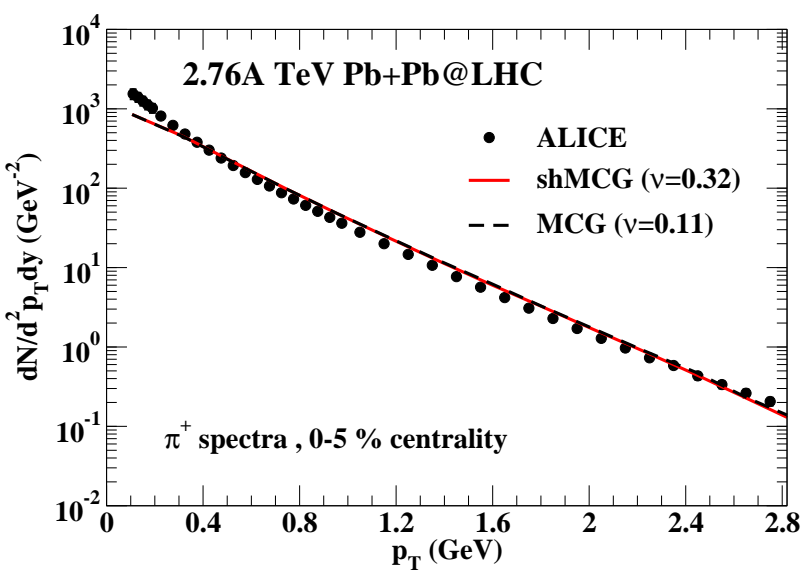

FIG. 8: (Color online) The thermal pion spectra evaluated for LHC with two types of initial conditions (see text) and comparison with ALICE data [46].

(Fig. 10(b)) collision conditions with (solid) and without (dashed) shadowing effects. We notice from the results displayed that the $v_{2}$ of $\pi^{+}$is enhanced by about $29 \%$ for shMCG initial condition compared to the $v_{2}$ obtained with MCG initial condition at $p_{T}=1.74 \mathrm{GeV}$ (around $p_{T}=1.74 \mathrm{GeV}, v_{2}$ of photons attains maximum) at RHIC energy. However, the elliptic flow of photons is about $36 \%$ more in shMCG than MCG initial condition at the same $p_{T}$ for RHIC energy. Similar behavior is observed at the LHC as well. This is indicative of the fact that the photons are able to capture the shadowing effects in the initial condition more effectively than hadrons. Also, it is shown in Ref. [4] that with event-by-event fluctuating initial condition the elliptic flow of photons is substantially enhanced. A detailed study is under progress on the elliptic flow of photons and hadrons with shadowing and event-by-event fluctuating initial condition to estimate the net enhancement in the $v_{2}$ of photons [43].

\section{SUMMARY AND CONCLUSIONS}

We have considered the effects of nucleon shadowing in the MC Glauber initial condition and calculated thermal photon spectra and elliptic flow for $200 \mathrm{~A} \mathrm{GeV} \mathrm{Au}+\mathrm{Au}$ collision at RHIC and $2.76 \mathrm{~A} \mathrm{TeV} \mathrm{Pb}+\mathrm{Pb}$ collision at LHC for $20-40 \%$ centrality bin. The initial conditions both for MCG and shMCG are constrained to the same experimentally measured charged hadron multiplicity for RHIC. Similar exercise has been repeated for LHC energies for MCG and shMCG initial conditions. Results without these constraints have also been shown. Relativistic hydrodynamic equations in $(2+1)$ dimensions have been solved with lattice QCD EoS to study the space time evolution of the system. We calculate photon spectra and elliptic flow considering both wounded nucleon as well as a two component model where the initial energy is taken to be proportional to a linear combination of $N_{\text {coll }}$ 

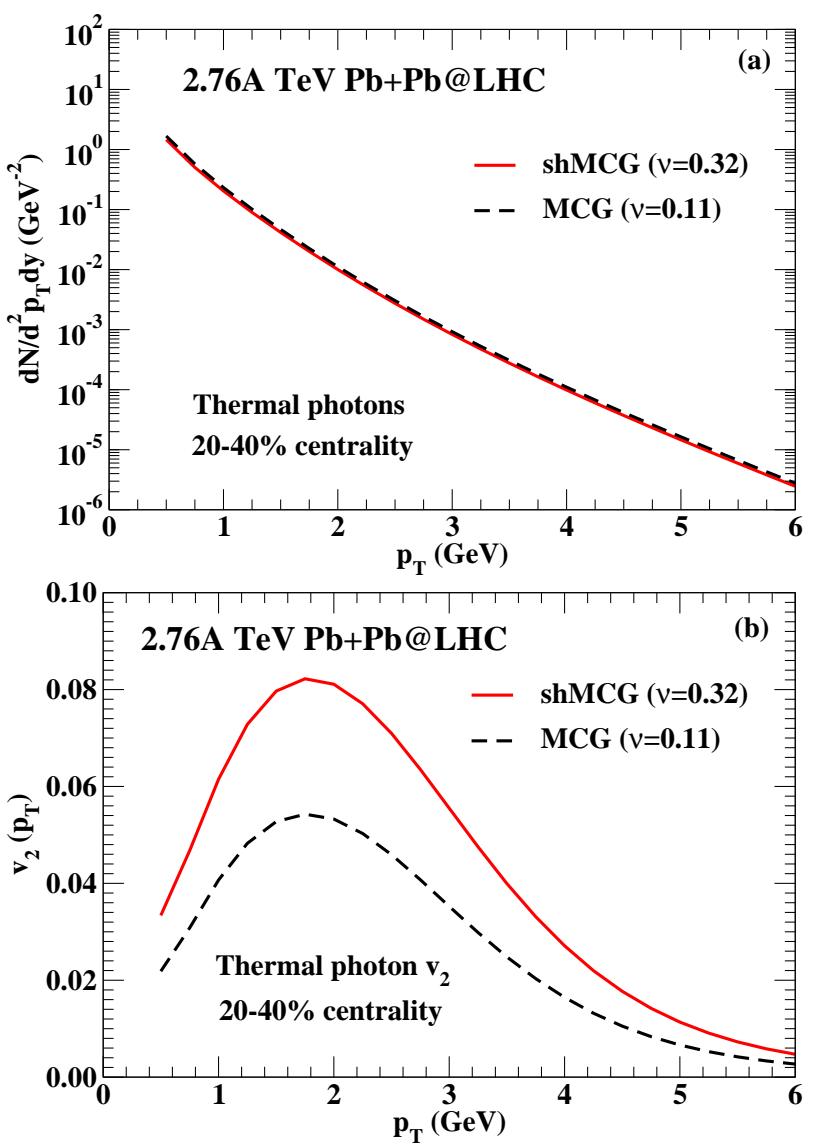

FIG. 9: (Color online) (a) The $p_{T}$ spectra and (b) $v_{2}$ of thermal photons for LHC energy with two types of initial conditions (see text).

and $N_{\text {part }}$. The results on the $p_{T}$ spectra of photons and pions both at RHIC and LHC collision conditions are found to be insensitive to the shadowing effects if the initial conditions are constrained to reproduce the same charged hadron multiplicity. However, the elliptic flow of thermal photons from shMCG initial condition is found to be significantly larger compared to the MCG initial condition as shadowing enhances the asymmetry by decreasing the effective ratio of minor to major axis of the elliptic overlap zone. Therefore, the shadowing effects have the potential to affect those quantities which depend on the geometric asymmetry of the system formed in HICRE. The effects of shadowing is found to be smaller in $v_{2}$ of hadrons than in the $v_{2}$ of photons. In this calculation, we consider initial state averaged smooth profile only which is expected to provide a qualitative picture of the effect of initial state shadowing on photons. Therefore, a complete calculation considering the initial state shadowing in event-by-event fluctuating initial condition would be useful to understand the $v_{2}$ of photons better, as it will then contain the contributions from both initial fluctuations as well as non-spherical geometry of the collision zone.
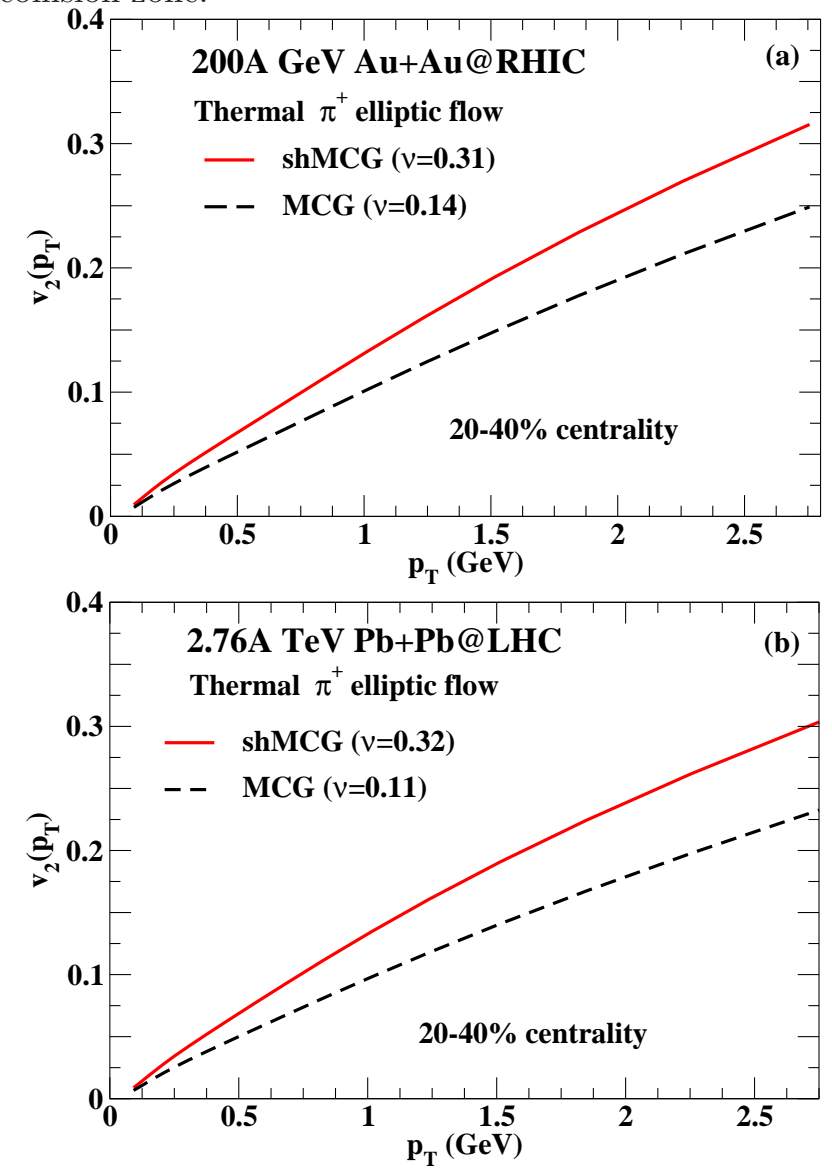

FIG. 10: (Color online) (a) The $v_{2}$ of thermal pions for RHIC (upper panel) and (b) LHC (lower panel) initial conditions (see text).

\section{ACKNOWLEDGEMENTS}

We thank C\&I group, VECC for providing computer facility and PDG is grateful to Department of Atomic Energy for financial support. Discussions with Dinesh Kumar Srivastava and Snigdha Ghosh are gratefully acknowledged.
[1] J. Alam, B. Sinha, and S. Raha, Phys. Rep. 273, 243 (1996)

[2] D. K. Srivastava, J. Phys. G 35, 104026 (2008).

[3] C. Gale, Y. Hidaka, S. Jeon, S. Lin, J.-F. Paquet, R. D. Pisarski, D. Satwo, V. V. Spkokov and G. Vujanovic,
Phys. Rev. Lett. 114, 072301 (2015).

[4] R. Chatterjee, H. Holopainen, I. Helenius, T. Renk, K. J. Eskola, Phys. Rev. C 88, 034901 (2013).

[5] C. Shen, U. Heinz, J.-F. Paquet, I. Kozlov and C. Gale, Phys. Rev. C 91, 024908 (2015). 
[6] A. Monnai, Phys. Rev. C 90, 021901 (2014).

[7] L. McLerran and B. Schenke, Nucl. Phys. A 929, 71 (2014).

[8] F.-M. Liu and S.-X. Liu, Phys. Rev. C 89, 034906 (2014).

[9] G. Basar, D. E. Kharzeev and V. Skokov, Phys. Rev. Lett. 109, 202303 (2012).

[10] K. Tuchin, Phys. Rev. C 87, 024912 (2013).

[11] B. G. Zakharov, Eur. Phys. J. C 76, 609 (2016).

[12] G.Vujanovic, J. F. Paquet, G. S. Denicol, M. Luzum, B. Schenke, S. Jeon and C. Gale, Nucl. Phys. A 932, 230 (2014).

[13] M. M. Aggarwal et al., [WA98 Collaboration], Phys. Rev. Lett. 85, 3595 (2000).

[14] A. Adare et al. (PHENIX Collaboration) Phys. Rev. Lett. 104, 132301 (2010), A. Adare et al. (PHENIX Collaboration), Phys. Rev. C 91, 064904 (2015).

[15] M. Wilde for the ALICE Collaboration, Nucl. Phys. A 904-905, 573c (2013); J. Adam et al. (ALICE Collaboration), Phys. Lett. B 754, 235 (2016).

[16] S. Ryu, J. F. Paquet, C. Shen, G. S. Denicol, B. Schenke, S. Jeon and C. Gale, Phys. Rev. Lett. 115, 132301 (2015).

[17] J. F. Paquet, C. Shen, G. S. Denicol, M. Luzum, B. Schenke, S. Jeon and C. Gale, Phys. Rev. C 93, 044906 (2016).

[18] H. van Hees, M. He and R. Rapp, Nucl. Phys. A 933, 256 (2015).

[19] H. van Hees, C. Gale and R. Rapp, Phys. Rev. C 84, 054906 (2011).

[20] O. Linyuk, W. Cassing and E.L. Bratkovskaya, Phys. Rev. C 89, 034908 (2014).

[21] A. Adare et al. (PHENIX Collaboration) Phys. Rev. Lett. 109, 122302 (2012); A. Adare et al. (PHENIX Collaboration) Phys. Rev. C 94, 064901 (2016).

[22] D. Lohner for the ALICE Collaboration, J. Phys. Conf. Ser. 446, 012028 (2013).

[23] http://cerncourier.com/cws/article/cern/63155.

[24] R. Chatterjee, H. Holopainen, T. Renk, and K. J. Eskola, Phys. Rev. C 83, 054908 (2011); R. Chatterjee, H. Holopainen, T. Renk, K. J. Eskola, J. Phys. G. Nucl. Part. Phys. 38, 124136 (2011).

[25] R. Chatterjee H. Holopainen, T. Renk, and K. J. Eskola, Phys. Rev. C 85, 064910 (2012); R. Chatterjee H. Holopainen, T. Renk, and K. J. Eskola, arXiv:1207.6917.

[26] S. Chatterjee, S. K. Singh, S. Ghosh, Md. Hasanujjaman,
J. Alam, and S. Sarkar, Phys. Lett. B 758, 269 (2016).

[27] S. Ghosh, S. K. Singh, S. Chatterjee, J. Alam, and S. Sarkar, Phys. Rev. C 93, 054904 (2016).

[28] H. Holopainen, H. Niemi, and K. Eskola, Phys. Rev. C 83, 034901 (2011).

[29] K. J. Eskola, K. Kajantie, P. V. Ruuskanen, and K. Tuominen, Nucl. Phys. B 570, 379 (2000).

[30] J. Kapusta, P. Lichard, and D. Seibert, Phys. Rev. D 44, 2774 (1991).

[31] R. Bair, H. Nakkagawa, A. Niegawa, and K. Redlich, Z. Phys. C 53, 433 (1992).

[32] E. Braaten and R. D. Pisarski, Nucl. Phys. B 337, 569 (1990); ibid 339, 310 (1990).

[33] P. Aurenche, F. Gelis, R. Kobes, and H. Zaraket, Phys. Rev. D 58, 085003 (1998).

[34] P. Arnold, G. D. Moore, and L.G. Yaffe, J. High Energy Phys. 0111, 057 (2001) ; P. Arnold, G.D. Moore, and L.G. Yaffe, J. High Energy Phys. 0112, 009 (2001) ; P. Arnold, G.D. Moore, and L.G. Yaffe, J. High Energy Phys. 0206, 030 (2002).

[35] J. Ghiglieri, J. Hong, A. Kurkela, E. Lu, G. D. Moore, and D. Teaney, JHEP 1305, 010 (2013).

[36] O. Kaczmarek and F. Zantow Phys. Rev D 71, 114510 (2005).

[37] S. Turbide, R. Rapp, and C. Gale, Phys. Rev. C 69, 014903 (2004).

[38] S. Sarkar, J. Alam, P. Roy, A. K. Dutt-Mazumder, B. Dutta-Roy and B. Sinha, Nucl. Phys. A 634, 206 (1998).

[39] P. Roy, S. Sarkar, J. Alam and B. Sinha, Nucl. Phys. A 653, 277 (1999).

[40] J. Alam, P. Roy and S. Sarkar Phys. Rev. C 71, 059802 (2005).

[41] M. Laine and Y. Schroder, Phys. Rev. D 73, 085009 (2006).

[42] R. Chatterjee, E. Frodermann, U. W. Heinz, and D. K. Srivastava, Phys. Rev. Lett. 96, 202302 (2006); R. Chatterjee and D. K. Srivastava, Phys. Rev. C 79, 021901(R) (2009); U. W. Heinz, R. Chatterjee, E. S. Frodermann, C. Gale, and D. K. Srivastava,

Nucl. Phys. A 783, 379 (2007).

[43] P. Dasgupta et. al. [in preparation].

[44] F. Cooper and G. Frye, Phys. Rev. D 10, 186 (1974).

[45] S. S. Adler et al., Phys. Rev. C 69, 034909 (2004).

[46] B. Abelev et al., Phys. Rev. C 88, 044910 (2013). 